\title{
Primary aldosteronism concurrent with subclinical Cushing's syndrome: a case report and review of the literature
}

\author{
Yingxiao Zhang ${ }^{1}$, Jianyu Tan ${ }^{1}$, Qin Yang ${ }^{1}$, Zhipeng Du', Shumin Yang ${ }^{1}$, Wenwen He${ }^{1}$, Ying Song ${ }^{1}$, Jinbo Hu', \\ Yi Yang ${ }^{1}$, Qifu Li ${ }^{1}$, Yao Zhang ${ }^{2}$, Yunfeng He ${ }^{2}$, Qingfeng Cheng ${ }^{1 *}$ (D) and the Chongqing Primary Aldosteronism \\ Study (CONPASS) Group
}

\begin{abstract}
Background: The prevalence of primary aldosteronism concurrent with subclinical Cushing's syndrome was higher than previously thought. Through analyzing a rare clinical case, we summarized the diagnosis and management of primary aldosteronism with subclinical Cushing's syndrome.

Case presentation: A 54-year-old Chinese man of Han nationality was diagnosed as having primary aldosteronism with subclinical Cushing's syndrome. An abdominal computed tomography scan revealed a mass in his left adrenal gland and a mass in his right adrenal gland. After finishing sequential adrenal venous sampling without adrenocorticotropic hormone, the result reminded us that the left and right nodules were responsible for hypercortisolism and aldosterone hypersecretion, respectively. Right and left adrenalectomy were performed successively. The pathological diagnosis was adrenocortical adenoma for both. Histological findings revealed that the right one had positive immunostaining for CYP11B2 and the left one had positive immunostaining for CYP11B1. The immunohistochemistry result helped us to confirm the diagnosis. Somatic KCNJ5 mutation (Leu168Arg) was found in the right tumor; there was no KCNJ5 mutation in the left adrenal tumor.

Conclusions: We suggest that patients with primary aldosteronism should have a low-dose overnight dexamethasone suppression test to screen for hypercortisolism. It can help avoid misdiagnoses and contribute to proper understanding of the adrenal vein sampling result. Making sure of the nidus of aldosterone and cortisol secretion is crucial for the therapy of patients with primary aldosteronism and subclinical Cushing's syndrome.
\end{abstract}

Keywords: Primary aldosteronism, Subclinical Cushing's syndrome, Adrenal vein sampling, Subtype

\section{Background}

Primary aldosteronism (PA) is a relatively common cause of secondary hypertension by excess aldosterone production from aldosterone-producing adenoma or idiopathic hyperaldosteronism in most cases [1]. Subclinical Cushing's syndrome (SCS) is a disease whose laboratory characteristics accord with Cushing's syndrome, but the typical clinical manifestation of Cushing's syndrome is absent [2]. The prevalence of PA concurrent with SCS is higher than previously

\footnotetext{
* Correspondence: cqf19760516@163.com

'Department of Endocrinology, The First Affiliated Hospital of Chongqing

Medical University, No.1 Youyi St., Chongqing 400016, China

Full list of author information is available at the end of the article
}

thought [3]. We present a rare case of bilateral adrenal tumors in which the left adrenocortical tumor produced cortisol and the right adrenocortical tumor secreted aldosterone, and we review literature on PA concurrent with SCS.

\section{Case presentation}

A 54-year-old Chinese man of Han nationality, a retiree, went to a local hospital because of hematuria. He had an abdominal computed tomography (CT) scan showing right renal mass and bilateral adrenal nodules. He was referred to our hospital for further examination and treatment. He denied any medical history, except for a 4-year history of hypertension.

(c) The Author(s). 2020 Open Access This article is distributed under the terms of the Creative Commons Attribution 4.0 International License (http://creativecommons.org/licenses/by/4.0/), which permits unrestricted use, distribution, and 
He took $5 \mathrm{mg}$ amlodipine besylate every day. He was married and living with his family; he had smoked tobacco for 30 years and denied alcohol consumption. Members of his family had no history of endocrine diseases or malignant tumors.

At the time of admission, his temperature was $37^{\circ} \mathrm{C}$, the pulse was 80 per minute, and his blood pressure was $161 / 75 \mathrm{mmHg}$. His height was $168 \mathrm{~cm}$ and weight $64 \mathrm{~kg}$. There was no physical sign of Cushing's syndrome, such as central obesity, skin atrophy, buffalo hump, red striae of skin, or moon face. The results of his cardiovascular, respiratory, abdominal, and neurological examinations were all unremarkable. A laboratory examination (Table 1) showed an electrolyte disturbance, in particular, a very low serum potassium level. His 24-hour urinary free

Table 1 Laboratory and endocrinological data

\begin{tabular}{ll}
\hline Test & Result \\
\hline Blood routine examination & \\
White blood cells & $6.46 \times 10^{9} / \mathrm{L}$ \\
Red blood cells & $5.14 \times 10^{12} / \mathrm{L}$ \\
Hemoglobin & $152 \mathrm{~g} / \mathrm{L}$ \\
Platelets & $224 \times 10^{9} / \mathrm{L}$ \\
Biochemistry & \\
Total protein & $68 \mathrm{~g} / \mathrm{L}$ \\
Albumin & $44 \mathrm{~g} / \mathrm{L}$ \\
Aspartate aminotransferase & $15 \mathrm{U} / \mathrm{L}$ \\
Alanine aminotransferase & $11 \mathrm{U} / \mathrm{L}$ \\
Blood urea nitrogen & $5 \mathrm{mmol} / \mathrm{L}$ \\
Creatinine & $86 \mathrm{umol} / \mathrm{L}$ \\
Na & $149 \mathrm{mmol} / \mathrm{L}$ \\
K & $1.9 \mathrm{mmol} / \mathrm{L}$ \\
Cl & $98 \mathrm{mmol} / \mathrm{L}$ \\
Total cholesterol & $3.91 \mathrm{mmol} / \mathrm{L}$ \\
Fasting blood glucose & $4.8 \mathrm{mmol} / \mathrm{L}$ \\
Urinary excretion & \\
Cortisol & $109.2 \mathrm{ng} / \mathrm{L}$ \\
Thunction of thyroid gland & \\
Fredothyronine & $2306.06 \mathrm{nmol} / 24 \mathrm{hours}$ \\
Mretane triiodothyronine & \\
\hline
\end{tabular}

cortisol was elevated. The function of his thyroid gland, parathyroid hormone, metanephrine, and normetanephrine were in normal range.

The endocrine findings (Table 2) showed high plasma aldosterone level and low plasma renin concentration. He then underwent a captopril challenge test which did not show suppressed level of plasma aldosterone concentration. A diagnosis of PA was of no doubt. On the other hand, the circadian rhythm of cortisol disappeared, and his serum cortisol level was not suppressed by an overnight dexamethasone suppression test (ODST) and low-dose dexamethasone suppression test (LDDST). He was diagnosed as having SCS.

An abdominal CT scan revealed a mass in the parenchyma of his right kidney (Figs. 1 and 2) and there was a rounded, low-density mass in his right adrenal gland and a rounded, low-density mass in his left adrenal gland (Figs. 3, 4, 5, and 6); the larger adrenal mass was approximately $2.6 \mathrm{~cm} \times 2.3 \mathrm{~cm}$ on the left adrenal gland. These findings indicated the possibility of a renal tumor and bilateral adrenocortical adenomas.

Sequential adrenal venous sampling (AVS) without adrenocorticotropic hormone (ACTH) stimulation was performed next. The result of AVS (Table 3) reminded us that the right adrenal gland was responsible for aldosterone hypersecretion, and, quite possibly, the left was responsible for hypercortisolism.

Right nephrectomy and right partial adrenalectomy were performed first. The golden-yellow nodule in the right adrenal was approximately $2.5 \mathrm{~cm} \times 2 \mathrm{~cm} \times 0.8 \mathrm{~cm}$ macroscopically. The pathological diagnosis of the right adrenal nodule was adrenocortical adenoma. The right kidney tumor was proven to be a fibroma. Three days after operation, our patient's serum potassium was 4.5 $\mathrm{mmol} / \mathrm{L}$ without any supplementary treatment. The hypertensive drug was changed to $2 \mathrm{mg}$ terazosin every day.

A laparoscopic left partial adrenalectomy was conducted 1 month later. When admitted to hospital, his blood pressure was $127 / 83 \mathrm{mmHg}$ controlled by $2 \mathrm{mg}$ terazosin every day, and his level of potassium was 4.9 $\mathrm{mmol} / \mathrm{L}$ without any supplementary treatment. We examined his cortisol at $8 \mathrm{a} . \mathrm{m}$. before operation, which was $344.81 \mathrm{nmol} / \mathrm{L}$. The operation was successful and the level of cortisol at 8 a.m. had reduced to $192.01 \mathrm{nmol} / \mathrm{L}$ 2 days after operation. The nodule in the left adrenal was golden-yellow; it was approximately $3 \mathrm{~cm} \times 2.5 \mathrm{~cm} \times$ $2 \mathrm{~cm}$. The pathological diagnosis of the left adrenal gland nodule was proven to be adrenocortical adenoma. After the second operation, doctors advised $30 \mathrm{mg}$ hydrocortisone should be taken once a day with a weekly reduction of $10 \mathrm{mg}$ until withdrawal, and $2 \mathrm{mg}$ terazosin should be taken once a day. 
Table 2 Endocrinological examinations

\begin{tabular}{lll}
\hline Examinations & & Result \\
\hline Endocrinology data & & \\
Plasma aldosterone concentration $(\mathrm{pg} / \mathrm{ml})$ & 454 & \\
Plasma renin concentration $(\mathrm{ulU} / \mathrm{ml})$ & 0.83 & 120 \\
Captopril loaded test & & 467 \\
Time (minutes) & 0 & 0.4 \\
Plasma aldosterone concentration $(\mathrm{pg} / \mathrm{ml})$ & 443 & $12: 00$ \\
Plasma renin concentration $(\mathrm{ulU} / \mathrm{ml})$ & 0.4 & 13.37 \\
Circadian rhythm & & 193.58 \\
Clock time & $8: 00$ & $24: 00$ \\
Adrenocorticotropic hormone $(\mathrm{pg} / \mathrm{ml})$ & 34.87 & 6.95 \\
Cortisol (nmol/L) & 524.32 & 166.65 \\
Dexamethasone suppression tests & & 72.16 \\
Dexamethasone & $1 \mathrm{mg}$ & \\
Cortisol (nmol/L) & 99.48 & \\
\hline
\end{tabular}

During postoperative follow-up, terazosin was gradually stopped, and blood pressure and serum potassium remained normal. Approximately 2 months later, his ACTH concentration was $98.73 \mathrm{pg} / \mathrm{ml}$ which was above the reference value. This meant our patient had adrenocortical insufficiency, and he continued to take hydrocortisone. Months later, hydrocortisone was stopped, the concentration of cortisol and ACTH were $233.46 \mathrm{nmol} / \mathrm{L}$ and $49.43 \mathrm{pg} / \mathrm{ml}$, respectively. The plasma aldosterone concentration was $104 \mathrm{pg} / \mathrm{ml}$ and plasma renin concentration was $3.1 \mathrm{uIU} / \mathrm{ml}$. Normalization of aldosterone-to-renin ratio (ARR),

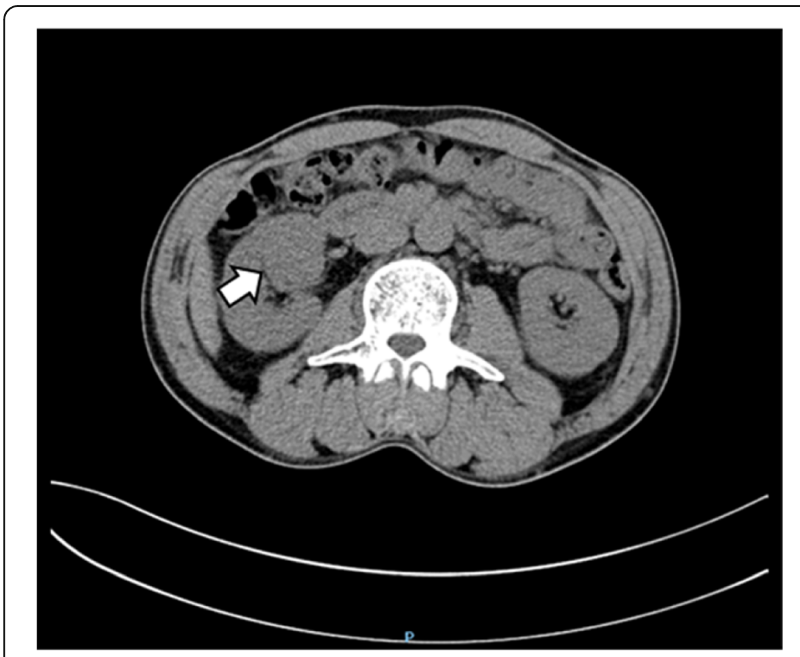

Fig. 1 Plain computed tomography scan shows mass in the right kidney. Arrow: kidney tumor cortisol, and ACTH were reached and our patient did not complain of any discomfort.

Immunohistochemistry (Fig. 7) of the two adrenal nodules revealed that the right one had positive immunostaining for CYP11B2, which is essential to synthesize aldosterone, and the left one had positive immunostaining for CYP11B1, which is essential to synthesize cortisol. These results confirmed the right nodule was responsible for the secretion of aldosterone and the left nodule was responsible for the secretion of cortisol. Somatic KCNJ5 mutation (Leu168Arg) was found in the right adrenal tumor

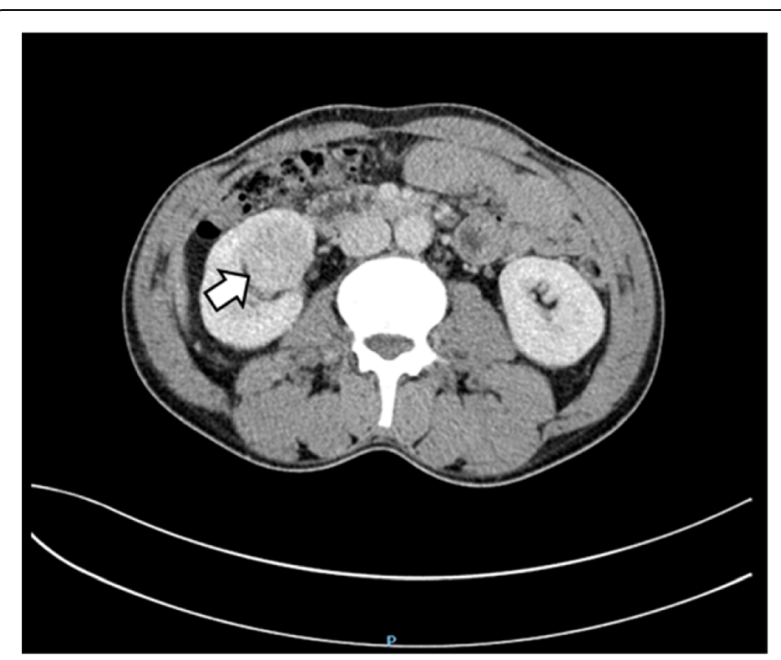

Fig. 2 Contrast computed tomography scan shows mass in the right kidney. Arrow: kidney tumor 


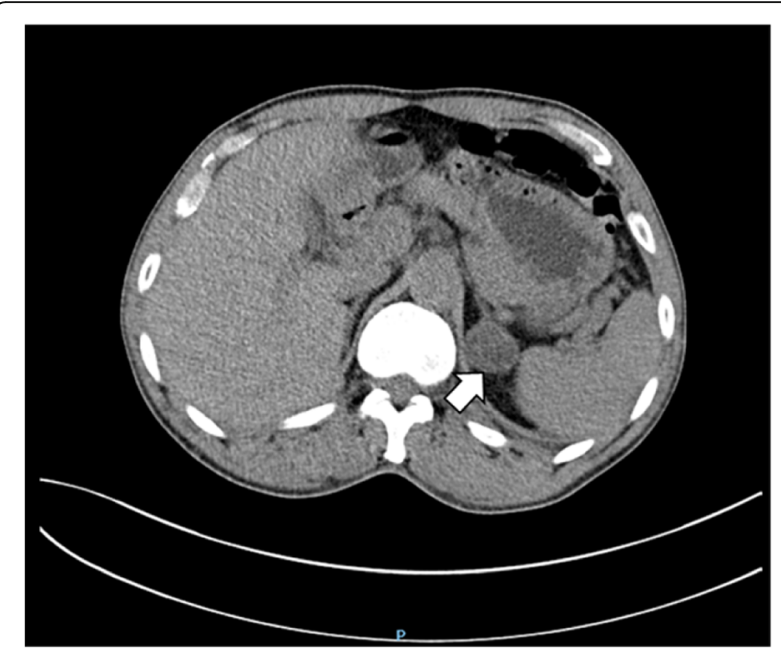

Fig. 3 Plain computed tomography scan shows mass in the left adrenal gland. Arrow: left adrenal tumor

(Fig. 8), and there was no KCNJ5 mutation in the left adrenal tumor.

\section{Discussion}

This was a rare case of bilateral adrenal tumors, in which the left adrenocortical tumor produced cortisol and the right one secreted aldosterone. The diagnosis was supported by endocrine findings before and after surgery and immunohistochemical evaluation of steroidogenic enzymes. We identified the type of mutation in two adenomas. However, there are some limitations to our study. The usual surgery management of aldosterone-producing adenoma is total adrenalectomy, but a partial adrenalectomy was conducted on our

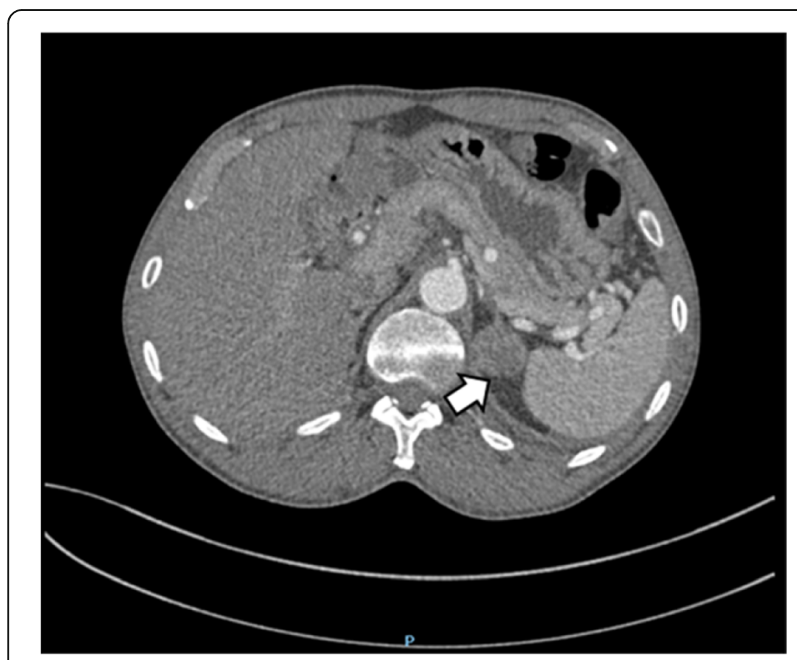

Fig. 4 Contrast computed tomography scan shows mass in the left adrenal gland. Arrow: left adrenal tumor

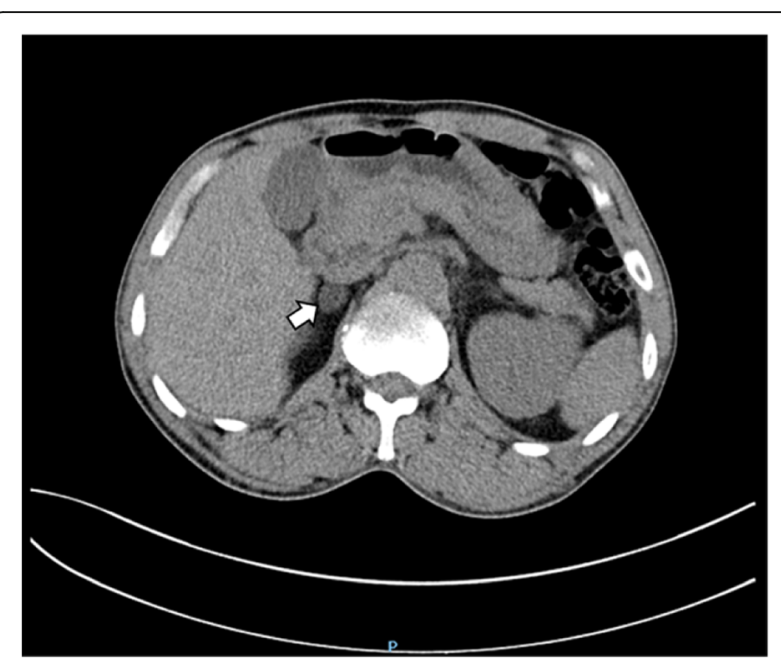

Fig. 5 Plain computed tomography scan shows mass in the right adrenal gland. Arrow: right adrenal tumor

patient because of the fear of adrenal crisis after surgery of both adrenal glands. We did not measure hormones such as metanephrine in the adrenal vein sample, making the interpretation of AVS difficult. And the follow-up visits were not perfect: we did not repeat dexamethasone suppression test and $1 \mathrm{mg}$ ODST after surgery.

Usually, cortisol and aldosterone are secreted by the same adenoma when endocrine findings indicate PA associated with SCS, and CT shows only one nodule in unilateral adrenal gland [4-8]. However, there is not always a single adenoma, so the result of CT can confuse our perception of the nidus of aldosterone or cortisol secretion. Hiraishi et al. classified PA

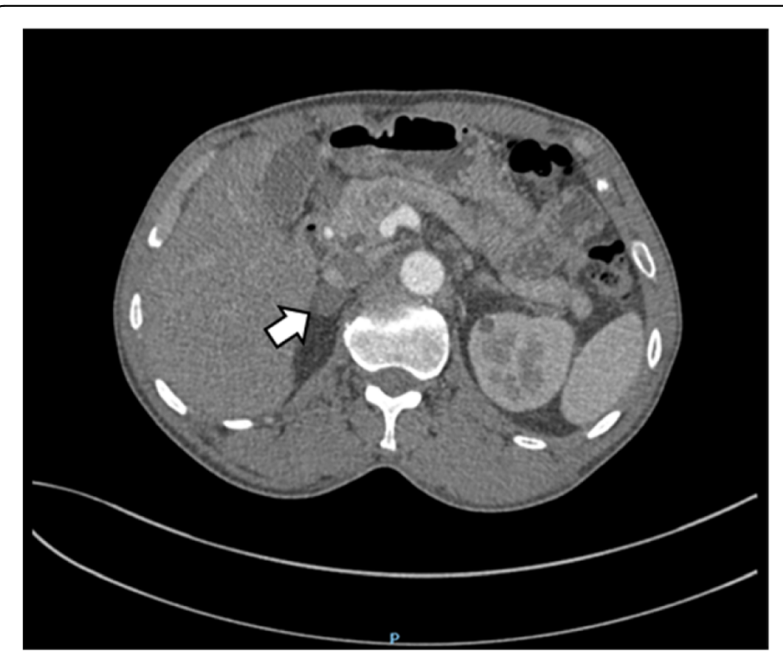

Fig. 6 Contrast computed tomography scan shows mass in the right adrenal gland. Arrow: right adrenal tumor 
Table 3 The result of adrenal vein sampling

\begin{tabular}{|c|c|c|c|c|}
\hline Sample & Aldosterone (pg/ml) & Cortisol (nmol/L) & Selectivity index & Aldosterone/cortisol ratio \\
\hline Left adrenal vein & 456.00 & 1268.42 & 5.63 & 0.36 \\
\hline Inferior vena cava & 420.00 & 225.10 & & 1.86 \\
\hline Right adrenal vein & $10,500.00$ & 294.49 & 1.78 & 35.65 \\
\hline Inferior vena cava & 336.00 & 165.54 & & 2.02 \\
\hline
\end{tabular}

Selectivity index: cortisol of adrenal vein/cortisol of inferior vena cava

associated with SCS by the result of CT, AVS, histology, and immunohistochemistry into six kinds of subtype derived from their eight cases [9]. Although not every case finished all the examinations, such as AVS or histology, some subtypes were speculated; these subtypes still shined a light on our confusion and reminded us that different therapeutic approaches may depend on the disease subtype.

The importance of AVS in the subtype diagnosis of PA is remarkable; AVS should also be performed in patients with PA associated with SCS, which may decide the therapeutic strategy. However, the study of Goupil et al. showed that concomitant adrenal autonomous cortisol and aldosterone secretion have potential to confound the lateralization of AVS [10]. The excess production of cortisol may lower the aldosterone/cortisol ratio on the side of the lesion while the opposite side increases due to contralateral cortisol suppression, thereby resulting in an incorrect diagnosis. Goupil et al. avoided the problem by measuring the concentration of metanephrine in the venous blood instead of cortisol [10]. They reported a case whose AVS result was bilateral aldosterone production by using aldosterone/cortisol ratio; however, the result of changing to aldosterone/metanephrine ratio showed aldosterone production lateralized to the left with right suppression. The patient underwent surgery and the case was pathologically proved. At last, the patient's hypertension improved and the PA and hypercortisolism were cured, which was confirmed by negative postoperative fludrocortisone suppression and ODST.

\section{Conclusion}

Due to the high prevalence of hypercortisolism in patients with PA, we suggest performing a low-dose
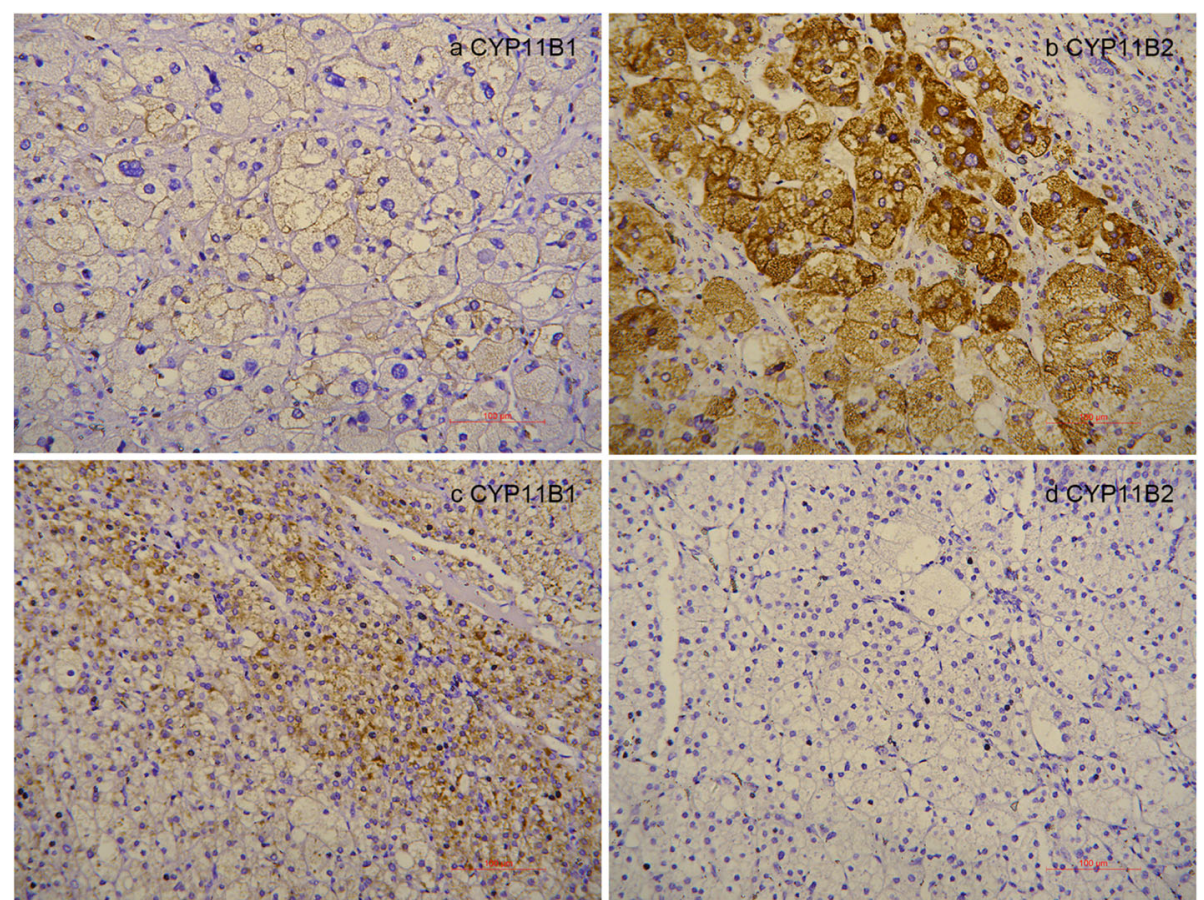

Fig. 7 CYP11B1 immunohistochemistry of right adrenal nodule (a). CYP11B2 immunohistochemistry of right adrenal nodule (b). CYP11B1 immunohistochemistry of left adrenal nodule (c). CYP11B2 immunohistochemistry of left adrenal nodule (d) 
right adrenal tumor

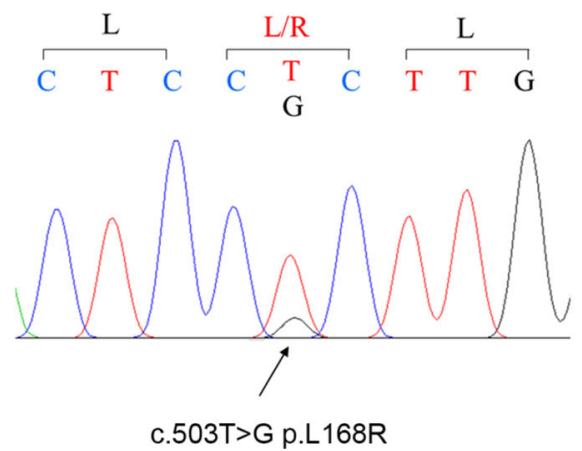

\section{left adrenal tumor}
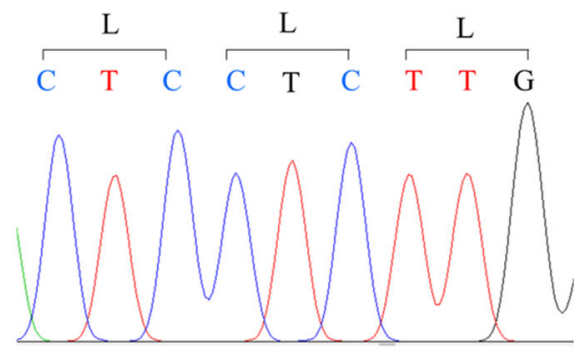

wild type

Fig. 8 The result of KCNJ5 DNA sequencing

overnight dexamethasone suppression test to screen hypercortisolism before surgery and AVS. If hypercortisolism is identified, hormones such as metanephrine in the adrenal vein sample should be measured. Not only can this prevent misunderstanding of the AVS result, but also help to avoid adrenal crisis after removal of the tumor.

\section{Abbreviations}

ACTH: Adrenocorticotropic hormone; ARR: Aldosterone-to-renin ratio; AVS: Adrenal venous sampling; CT: Computed tomography; LDDST: Low-dose dexamethasone suppression test; ODST: Overnight dexamethasone suppression test; PA: Primary aldosteronism;

SCS: Subclinical Cushing's syndrome

\section{Acknowledgements}

We are grateful to the membership of the Chongqing Primary Aldosteronism Study (CONPASS) Group.

\section{Authors' contributions}

QC and QL contributed to the conception of the work. YZ, JT, and QY contributed to the acquisition, analysis of data, and writing of manuscript. QC, ZD, and SY contributed to the conduct of adrenal venous sampling and interpretation of data. WH, YS, and JH contributed to the follow-up visit. YY contributed to the work of immunohistochemistry and sequencing. $Y Z$ and $\mathrm{YH}$ contributed to the operation. All authors read and approved the final manuscript.

\section{Funding}

This research received no specific grant from any funding agency in the public, commercial or not-for-profit sectors.

\section{Availability of data and materials}

The datasets analyzed during the current study are available from the corresponding author on reasonable request.

\section{Ethics approval and consent to participate}

Ethical Committee of the First Affiliated Hospital of Chongqing Medical University approved this study.

\section{Consent for publication}

Written informed consent was obtained from the patient for the publication of this case report and any accompanying images. A copy of the written consent is available for review by the Editor-in-Chief of this journal.

\section{Competing interests}

The authors declare that they have no competing interests.

\section{Author details}

'Department of Endocrinology, The First Affiliated Hospital of Chongqing Medical University, No.1 Youyi St., Chongqing 400016, China. ²Department of Urology, The First Affiliated Hospital of Chongqing Medical University, No.1

Youyi St., Chongqing 400016, China.

Received: 16 July 2019 Accepted: 21 January 2020

Published online: 20 February 2020

\section{References}

1. Funder JW, Carey RM, Mantero F, Murad MH, Reincke M, Shibata H, Stowasser M, Young WF. The management of primary aldosteronism: case detection, diagnosis, and treatment: an endocrine society clinical practice guideline. J Clin Endocrinol Metab. 2016;101(5):1889-916. https://doi.org/10. 1210/jc.2015-4061.

2. Stewart PM. Is subclinical Cushing's syndrome an entity or a statistical fallout from diagnostic testing? Consensus surrounding the diagnosis is required before optimal treatment can be defined. J Clin Endocrinol Metab. 2010;95(6):2618-20. https://doi.org/10.1210/jc.2010-0633.

3. Fallo F, Castellano I, Gomez-Sanchez CE, Rhayem Y, Pilon C, Vicennati V, Santini D, Maffeis V, Fassina A, Mulatero P, Beuschlein F, Reincke M. Histopathological and genetic characterization of aldosterone-producing adenomas with concurrent subclinical cortisol hypersecretion: a case series. Endocrine. 2017;58(3):503-12. https://doi.org/10.1007/s12020-0171295-4.

4. Adachi J, Hirai Y, Terui K, Nakano T, Fukuda Y, Suda T, Sasano H. A report of 7 cases of adrenal tumors secreting both cortisol and aldosterone. Intern Med. 2003;42(8):714-8. https://doi.org/10.2169/ internalmedicine.42.714.

5. Honda T, Nakamura T, Saito Y, Ohyama Y, Sumino H, Kurabayashi M. Combined primary aldosteronism and preclinical Cushing's syndrome: an unusual case presentation of adrenal adenoma. Hypertens Res. 2001;24(6): 723-6. https://doi.org/10.1291/hypres.24.723.

6. Makino S, Oda S, Saka T, Yasukawa M, Komatsu F, Sasano H. A case of aldosterone-producing adrenocortical adenoma associated with preclinical Cushing's syndrome and hypersecretion of parathyroid hormone. Endocr J. 2001;48(1):103-11. https://doi.org/10.1507/endocr.48.103.

7. Yamakita N, Murai T, Miyamoto K, Matsunami H, Ikeda T, Sasano H, Mune T, Yasuda K. Variant of pre-clinical Cushing's syndrome: hypertension and hypokalemia associated with normoreninemic normoaldosteronism. Hypertens Res. 2002;25(4):623-30. https://doi.org/ 10.1291/hypres.25.623.

8. Hogan MJ, Schambelan M, Biglieri EG. Concurrent hypercortisolism and hypermineralocorticoidism. Am J Med. 1977;62(5):777-82. https://doi.org/10. 1016/0002-9343(77)90883-x.

9. Hiraishi K, Yoshimoto T, Tsuchiya K, Minami I, Doi M, Izumiyama H, Sasano $\mathrm{H}$, Hirata Y. Clinicopathological features of primary aldosteronism associated with subclinical Cushing's syndrome. Endocr J. 2011;58(7):543-51. https:// doi.org/10.1507/endocrj.K10E-402. 
10. Goupil R, Wolley M, Ungerer J, McWhinney B, Mukai K, Naruse M, Gordon $\mathrm{RD}$, Stowasser M. Use of plasma metanephrine to aid adrenal venous sampling in combined aldosterone and cortisol over-secretion. Endocrinol Diabetes Metab Case Rep. 2015;2015:150075. https://doi.org/10.1530/edm15-007.

\section{Publisher's Note}

Springer Nature remains neutral with regard to jurisdictional claims in published maps and institutional affiliations.

- fast, convenient online submission

- thorough peer review by experienced researchers in your field

- rapid publication on acceptance

- support for research data, including large and complex data types

- gold Open Access which fosters wider collaboration and increased citations

- maximum visibility for your research: over $100 \mathrm{M}$ website views per year

At BMC, research is always in progress. 